\title{
Non-Minimally Coupled Cosmology as Geodesic Motion
}

\author{
Luciana A. Elias \\ Departamento de Matemática Aplicada, UNICAMP \\ C.P. 6065, 13083-859 - Campinas, SP, Brazil \\ E-mail: luelias@ime.unicamp.br
}

\author{
Alberto Saa*广 \\ Departamento de Matemática Aplicada, UNICAMP \\ C.P. 6065, 13083-859 - Campinas, SP, Brazil \\ E-mail: asaa@ime.unicamp.br
}

\begin{abstract}
Recent works showing that homogeneous and isotropic cosmologies involving scalar fields correspond to geodesics of certain augmented spaces are generalized to the non-minimal coupling case. As the Maupertuis-Jacobi principle in classical mechanics, this result allows us, in principle, to infer some of the dynamical properties of the cosmologies from the geometry of the associated augmented spaces.
\end{abstract}

Fifth International Conference on Mathematical Methods in Physics - IC2006 April 24-28 2006

Centro Brasilerio de Pesquisas Fisicas, Rio de Janeiro, Brazil

\footnotetext{
* Speaker.

† Corresponding author.
} 


\section{Introduction}

The Maupertuis-Jacobi principle[1] in classical mechanics establishes that the dynamics of a given system can be viewed as geodesic motions in an associated Riemannian manifold. Recalling briefly, let us consider a classical mechanical system with $N$ degrees of freedom described by the Lagrangian

$$
L(q, \dot{q})=\frac{1}{2} g_{i j}(q) \dot{q}^{i} \dot{q}^{j}-V(q),
$$

where $i, j=1,2, \ldots, N$, the dot stands for differentiation with respect to the time $t$, and $g_{i j}$ is the Riemannian metric on an $N$-dimensional configuration space $\mathscr{M}$. All the quantities here are assumed to be smooth. The Euler-Lagrange equations of (1.1) can be written as

$$
\ddot{q}^{i}+\Gamma_{j k}^{i} \dot{q}^{j} \dot{q}^{k}=-g^{i j} \partial_{j} V(q),
$$

where $\Gamma_{j k}^{i}$ is the Levi-Civita connection for the metric $g_{i j}$.

The Hamiltonian of the system described by (1.1)

$$
H(q, p)=\frac{1}{2} g^{i j}(q) p_{i} p_{j}+V(q),
$$

with $p_{i}=g_{i j} \dot{q}^{j}$, is obviously a constant of motion, namely the total energy. For a fixed energy $E$, the trajectories in the $2 N$-dimensional phase-space $\left(q^{i} ; p_{j}\right)$ are confined to the hypersurface $E=$ $\frac{1}{2} g^{i j} p^{i} p^{j}+V(q)$. On the other hand, the admissible region for the trajectories in the configuration space is given by

$$
\mathscr{D}_{E}=\{q \in \mathscr{M}: V(q) \leq E\} .
$$

In general, the region $\mathscr{D}_{E}$ can be bounded or not, connected or not. The boundary of the admissible region for the trajectories is given by

$$
\partial \mathscr{D}_{E}=\{q \in \mathscr{M}: V(q)=E\} .
$$

If the potential has no critical points on the boundary $(V \neq 0)$, then $\partial \mathscr{D}_{E}$ is a $N-1$ dimensional submanifold of $\mathscr{M}$. We can easily see that if a trajectory reaches the boundary $\partial \mathscr{D}_{E}$ at a point $q_{0}$, its velocity at this point vanishes and the trajectory approach or depart from $q_{0}$ perpendicularly to the boundary $\partial \mathscr{D}_{E}$. In particular, there is no allowed trajectory along the boundary.

One can show that the equations of motion (1.2) are, in the interior of $\mathscr{D}_{E}$, fully equivalent to the geodesic equation of the "effective" Riemannian geometry on $\mathscr{M}$ defined from the Jacobi metric[1]

$$
\hat{g}_{i j}(q)=2(E-V(q)) g_{i j}(q) .
$$

The geodesic equation in question is given by

$$
\hat{\nabla}_{u} u=\frac{d^{2} q^{i}}{d s^{2}}+\hat{\Gamma}_{j k}^{i} \frac{d q^{j}}{d s} \frac{d q^{k}}{d s}=0
$$

where $u=d q^{i} / d s$ is the tangent vector along the geodesic and $\hat{\nabla}$ and $\hat{\Gamma}_{j k}^{i}$ are, respectively, the covariant derivative and the Levi-Civita connection for the Jacobi metric $\hat{g}_{i j}$, and $s$ a parameter along the geodesic obeying

$$
\frac{d s}{d t}=2(E-V(q))
$$


As any classical topic, there is a vast literature on the Maupertuis-Jacobi principle. We notice only that, motivated by the celebrated result due to Anosov[2] stating that the geodesic flow in a compact manifold with all sectional curvatures negative at every point is chaotic, the MaupertuisJacobi principle has been recently invoked for the study of chaotic dynamics. (See, for instance, [3] and the references therein).

The main motivation of the present work is the the result presented in [4]. The authors considered cosmological models with $N$ self-interacting scalar fields $\phi^{\alpha}$ taking their values in a Riemannian target space endowed with a metric $G_{\alpha \beta}$. The corresponding actions is

$$
\int d^{4} x \sqrt{-g}\left(R-g^{i j} G_{\alpha \beta}(\phi) \partial_{i} \phi^{\alpha} \partial_{j} \phi^{\beta}-2 V(\phi)\right) .
$$

Greek indices run over $1 \ldots N$ (the target space dimension), while the lower case roman ones run over $1 \ldots 4$ (the spacetime dimension). The spacetime metric is $g_{i j}$ and $R$ stands for its scalar curvature. By considering the Friedman-Robertson-Walker homogeneous and isotropic metric

$$
d s^{2}=-d t^{2}+a^{2}(t) d \Sigma_{\kappa}^{2},
$$

$a(t)>0$, where $\Sigma_{\kappa}$ represents the 3-dimensional spatial sections of constant curvature $\kappa$, they showed, by using arguments close to the Maupertuis-Jacobi principle, that the equations of motion associated to the action (1.9) do indeed correspond to the geodesics of a certain "effective Jacobi" (pseudo) metric on an augment Lorentzian space. For the spatially flat case $(\kappa=0)$, for instance, the augmented space has $(1, N)$ signature and the geodesics corresponding to the equations of motion derived from (1.9) are timelike, null, or spacelike according, respectively, if $V>0, V=0$, or $V<0$. These results have been applied to the dynamical study of the models governed by actions of the type (1.9), see [5].

Applications of the Maupertuis-Jacobi principle to the field equations obtained from HilbertEinstein like actions have also a long history. Non-homogeneous and anisotropic cases were considered in [6]. Applications involving distinct differential spaces instead of differential manifolds were discussed in [7]. Non-minimally coupled scalar fields, however, have not been considered so far.

\section{A Maupertuis-Jacobi principle for non-minimally coupled cosmology}

Non-minimally coupled scalar fields are quite common in cosmology. In particular, they have been invoked recently to describe dark energy (see [8] and [9], and the references therein, for, respectively, models using conformal coupling and more general ones). The non-minimal coupled generalization of (1.9) we consider here is

$$
\int d^{4} x \sqrt{-g}\left(F(\phi) R-g^{i j} G_{\alpha \beta}(\phi) \partial_{i} \phi^{\alpha} \partial_{j} \phi^{\beta}-2 V(\phi)\right) .
$$

In this work, we restrict ourselves to the $\kappa=0$ case, the full analysis will appear elsewhere[10]. For such a case, one has $R=6 \dot{H}+12 H^{2}, H=\dot{a} / a$. Integrating by parts the action (2.1), we obtain the following Lagrangian

$$
L\left(a, \dot{a}, \phi_{\alpha}, \dot{\phi}^{\alpha}\right)=a^{3}\left(-6 H^{2} F-6 H \dot{\phi}^{\alpha} \partial_{\alpha} F+G_{\alpha \beta}(\phi) \dot{\phi}^{\alpha} \dot{\phi}^{\beta}-2 V(\phi)\right)
$$


for the system on the $N+1$ configuration space spanned by $\left(a, \phi_{\alpha}\right)$. By introducing the following Lorentzian metric

$$
G_{A B}\left(a, \phi^{\alpha}\right)=\left(\begin{array}{c:c}
-6 a F & -3 a^{2} \partial_{\beta} F \\
\hdashline-3 a^{2} \partial_{\alpha} F & a^{3} G_{\alpha \beta}
\end{array}\right)
$$

on the configuration space (upper case roman indices run over $0 \ldots N$ ), the Lagrangian (2.2) can be cast in the form

$$
L\left(\phi_{A}, \dot{\phi}^{A}\right)=G_{A B}(\phi) \dot{\phi}^{A} \dot{\phi}^{B}-2 V_{\mathrm{eff}}(\phi),
$$

where $\phi^{A}=\left(a, \phi^{\alpha}\right)$ and $V_{\text {eff }}\left(\phi^{A}\right)=a^{3} V\left(\phi^{\alpha}\right)$. It is clear the similarity with (1.1), provided that $\operatorname{det} G_{A B} \neq 0$, which we assume by now. We will return to this issue in the last Section.

Before considering the Maupertuis-Jacobi principle, let us recall that our manipulations imply that all solutions of the Euler-Lagrange of (2.1) are also solutions of the Euler-Lagrange equations of (2.2), but not the converse. Einstein equations form a constrained system. The solutions of (2.1) correspond, indeed, to a subset of the solutions of (2.2), as one can realize by considering the Hamiltonian associated to (2.2)

$$
H\left(\phi_{A}, \pi_{A}\right)=G^{A B} \pi_{A} \pi_{B}+2 V_{\mathrm{eff}}(\phi)=a^{3}\left(-6 H^{2} F-6 H \dot{\phi}^{\alpha} \partial_{\alpha} F+G_{\alpha \beta}(\phi) \dot{\phi}^{\alpha} \dot{\phi}^{\beta}+2 V(\phi)\right),
$$

which, obviously, must be a constant of motion, say $H\left(\phi_{A}, \pi_{A}\right)=E$. The Euler-Lagrange equations of (2.1), on the other hand, implies that $E=0$ (the so-called energy constraint). Hence, we must bear in mind that the relevant solutions of our original problem correspond indeed to the $E=0$ subset of the dynamics governed by (2.2). Now, let us consider separately the cases $V=0, V<0$ and $V>0$.

According to (1.2), for $V=0$ the Euler-Lagrange equations of (2.1) are already given by geodesics of (2.3). From the energy constraint

$$
G_{A B}(\phi) \dot{\phi}^{A} \dot{\phi}^{B}=-2 a^{3} V(\phi),
$$

one sees that such geodesics are of null type. For $V<0$, one can introduce the metric

$$
\hat{G}_{A B}=-2 V G_{A B}
$$

and obtain the same conclusions by repeating the same procedure used for the classical MaupertuisJacobi principle. From the energy constraint (2.6), it is clear that the associated geodesics will be spacelike. Finally, for $V>0$, one can introduce

$$
\hat{G}_{A B}=2 V G_{A B}
$$

and repeat exactly the same steps followed for the last case. In this case, the geodesics will be timelike. Since the metric (2.3) is not positive defined, one cannot, in general, obtain from the energy constraint a dynamically admissible region $\mathscr{D}$ of the configuration space, as done for the classical Maupertuis-Jacobi principle. Also as a consequence of the Lorentzian signature of (2.3), one has that, typically, $\mathscr{D}$ will be unbounded.

Summarizing, the equations governing the cosmological model (2.1) correspond to the geodesics of the metric

$$
\hat{G}_{A B}=\left\{\begin{array}{l}
G_{A B} \quad \text { if } V=0, \\
2|V| G_{A B} \quad \text { if } V>0 \text { or } V<0,
\end{array}\right.
$$


with $G_{A B}$ is given by (2.3). Moreover, since

$$
\hat{G}_{A B}(\phi) \dot{\phi}^{A} \dot{\phi}^{B}=-4 a^{3}|V| V,
$$

one has that the geodesics are of null type, timelike or spacelike, respectively, according if $V=0$, $V>0$, or $V<0$. All the results[4] that have motivated this work can be obtained by setting $F=1$.

\section{Final Remarks}

Since the equations for cosmological models like (2.1) correspond to the geodesic equations of the Lorentzian metric (2.9), it is possible, in principle, to obtain some information about the cosmological dynamics from the geometry of the associated Lorentzian metric. For instance, it is shown in [4] that cosmological solutions exhibiting late time acceleration are related to geodesics entering in a certain region corresponding to a subset of the lightcone of (2.9). The study of dynamical singularities can also benefit from these results. The essential assumption of $\operatorname{det} G_{A B} \neq 0$ is strictly related to the avoidance of some singularities. For the $N=1$ and $G_{\alpha \beta}=1$ case, one has

$$
\operatorname{det} G_{A B}=-6 a^{4}\left(F(\phi)+\frac{3}{2}\left(F^{\prime}(\phi)\right)^{2}\right) .
$$

The vanishing of this quantity is known to be associated with the existence of some unavoidable dynamical singularities (see, for references, [11]), which render the associated cosmological model unphysical. Some preliminary results suggest that the same occurs for the $N$-field case.

We finish by noticing that one of the most interesting peculiarities of the conformal coupling $\left(F(\phi)=1-\phi^{2} / 6\right)$ is that it can evade the $\operatorname{det} G_{A B}=0$ singularity, since

$$
F(\phi)+\frac{3}{2}\left(F^{\prime}(\phi)\right)^{2}=1
$$

for the conformal coupling and, consequently, the Maupertuis-Jacobi principle can be always employed.

\section{Acknowledgments}

The authors thank Dr. Ricardo Mosna for valuable help, and FAPESP and CNPq for the financial support.

\section{References}

[1] V.I. Arnold, Mathematical Methods of Classical Mechanics, Springer, New York, 1989.

[2] V.I. Arnold and A. Avez, Problèmes ergodiques de la Mécanique Classique, Gauthier-Villars, Paris, 1967.

[3] V.V. Kozlov, Symmetries, Topology and Resonances in Hamiltonian Mechanics, Springer, New York, 1996; J. Zsczęsny and T. Dobrowolski, Geodesic Deviation Equation Approach to Chaos, Ann. Phys. (N.Y.) 277, 161 (1999); A. Saa, On the viability of local criteria for chaos, ibid., 314, 508 (2004). 
[4] P.K. Townsend and M.N.R. Wohlfarth, Cosmology as geodesic motion, Class. Quantum Grav. 21, 5375 (2004).

[5] J.G. Russo and P.K. Townsend, Cosmology as relativistic particle mechanics: from big crunch to big bang, Class. Quantum Grav. 22, 737 (2005); Q. Guo, R.G Cai, Classification of cosmological trajectories, gr-qc/0504033.

[6] L. Smolin and C. Soo, The Chern-Simons invariant as the natural time variable for classical and quantum cosmology, Nucl. Phys. B449, 289 (1995); J. Greensite, Field theory as free fall, Class. Quantum Grav. 13, 1339 (1996); A. Carlini and J. Greensite, The mass shell of the universe, Phys. Rev. D55, 3514 (1997).

[7] M. Szydłowski, M. Heller, and W. Sasin, Geometry of spaces with the Jacobi metric, J. Math. Phys. 37 (1996), 346.

[8] S.S. Bayin, F.I. Cooperstock, V. Faraoni, A singularity-free cosmological model with a conformally coupled scalar field, Astrophys. J. 428, 439 (1994); V. Faraoni, Inflation and quintessence with nonminimal coupling, Phys. Rev. D62, 023504 (2000); E. Gunzig et al., Superinflation, quintessence, and nonsingular cosmologies, Phys. Rev. D63, 067301 (2001).

[9] V. Faraoni, Coupled oscillators as models of phantom and scalar field cosmologies, Phys. Rev. D69, 123520 (2004); F.C. Carvalho and A. Saa, Non-minimal coupling, exponential potentials and the $w<-1$ regime of dark energy, Phys. Rev. D70, 087302 (2004).

[10] L.A. Elias and A. Saa, Non-minimally coupled cosmologies and the Maupertuis-Jacobi principle, to appear.

[11] L.R Abramo, L Brenig, E Gunzig, A Saa, On the singularities of gravity in the presence of nonminimally coupled scalar fields, Phys. Rev. D67, 027301 (2003); Dynamical study of the singularities of gravity in the presence of nonminimally coupled scalar fields, Int. J. Theor. Phys. 42, 1145 (2003). 